\title{
Quantitative Magnetic Imaging of Skyrmions and Magnetic Thin Layers by Lorentz Transmission Electron Microscopy and Electron Holography
}

\author{
Toshiaki Tanigaki and Tetsuya Akashi
}

Research \& Development Group, Hitachi, Ltd., Hatoyama, Japan

Electron microscopy is advantageous for observing magnetic structures inside materials at high resolutions. There are several techniques in electron microscopy such as the Lorentz transmission electron microscopy (TEM), electron holography, differential phase contrast, diffractive imaging, and ptychography. Lorentz TEM has the easiest operations, requiring only a change in focus. Also, quantitative imaging in Lorentz TEM can be performed by using transport intensity equation (TIE). Electron holography has advantages with quantitative phase measurements and high resolution observation. Quantitative magnetic imaging by Lorentz TEM and electron holography is applicable for observing magnetic field distributions in magnetic skyrmions and thin layers.

Magnetic skyrmions are nanoscale magnetic structures stabilized topologically. The structures and currently-induced dynamics of skyrmions are hot topics, because skyrmions are a candidate for future memory devices. First, real-space observation of skyrmions has been performed by using Lorentz TEM [1]. A Bloch-type structure was developed by using TIE. The size of $\mathrm{Fe}_{0.5} \mathrm{Co}_{0.5} \mathrm{Si}$ was $90 \mathrm{~nm}$. Lorentz TEM was applied to observe the skyrmions in $\mathrm{MnSi}$ and a skyrmion lattice with $18 \mathrm{~nm}$ spacing was captured [2]. Lorentz TEM was also used to observe smaller skyrmions in MnGe. MnGe had a cubic lattice of skyrmions. Skyrmion lattices along the crystal lattice plane (100) with lattice spacing of $3 \mathrm{~nm}$ was observed [3]. Lorentz TEM combined with TIE successfully observed skyrmion structures at a nanometer scale. Lorentz TEM was also useful for observing skyrmion dynamics. The currently-induced dynamics of a coexistent state of magnetic skyrmions and helical magnetic structures were also investigated with the Lorentz TEM [4].

Electron holography has also been used to investigate three dimensional (3D) structures of skyrmions. The phase shift of electrons due to skyrmions in $\mathrm{Fe}_{0.5} \mathrm{Co}_{0.5} \mathrm{Si}$ as a function of the sample thickness were measured by using electron holography. For a sample of more than $300 \mathrm{~nm}$ thickness, a 1-MV holography electron microscope was used. The phase shift was linear to the sample thickness. This result indicated that the magnetic structure of the skyrmions in the sample was cylindrical [5]. To visualize the full 3D magnetic vector configurations, holographic tomography was needed. We successfully created a 3D reconstruction of the magnetic vortex structures in ferromagnetic discs [6], although they were not skyrmions.

To improve the resolution of the electron holography, we developed an aberration corrected 1.2-MV holography electron microscope. The spatial resolution of the developed microscope was $0.24 \mathrm{~nm}$ in field-free mode for observing magnetic samples. However, there were difficulties with the subnanometer resolution magnetic phase observation due to technical problems in separating the electrostatic and magnetic phases at high resolutions. To overcome this, we developed a pulse magnetization system. The system was used to reverse the magnetization of the sample without changing geometrical configurations in the microscope. A multilayer of $\mathrm{CoFeB} / \mathrm{Ta}$ was observed, and a spatial resolution of $0.67 \mathrm{~nm}$ was attained in the phase image and Fourier transform pattern [7]. 
Lorentz TEM and electron holography are useful techniques for quantitative imaging of magnetic structures at high resolutions. Tomographic approaches can visualize magnetic structures threedimensionally. Aberration corrected electron holography can further improve spatial resolution in magnetic phase imaging, if the electrostatic and magnetic phases are properly separated.

\section{References:}

[1] X. Yu et al., Nature 465 (2010), p. 901.

[2] A. Tonomura et al., Nano Lett. 12 (2012), p. 1673.

[3] T. Tanigaki et al., Nano Lett. 15 (2015), p. 5438.

[4] K. Shibata et al., Nano Lett. DOI: 10.1021/acs.nanolett.7b04312

[5] H.S. Park et al., Nat. Nanotech. 9 (2014), p. 337.

[6] T. Tanigaki et al., Nano Lett., 15 (2015), p. 1309.

[7] T. Tanigaki et al., Sci. Rep. 7 (2017), p. 16598.

[8] Development of the 1.2-MV holography microscope was supported by a grant from the Japan Society for the Promotion of Science (JSPS) through the "Funding Program for World-Leading Innovative R\&D on Science and Technology (FIRST Program)" initiated by the Council for Science, Technology, and Innovation (CSTI). Part of this research was supported by CREST, JST.

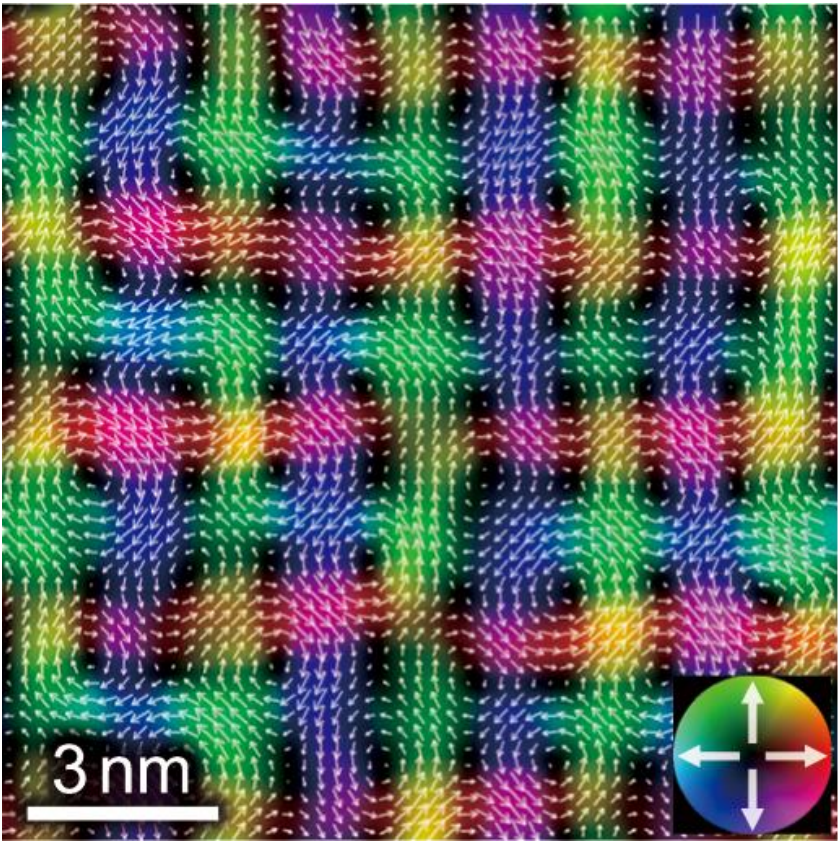

Figure 1. Magnetic skyrmion in MnGe observed by Lorentz TEM.

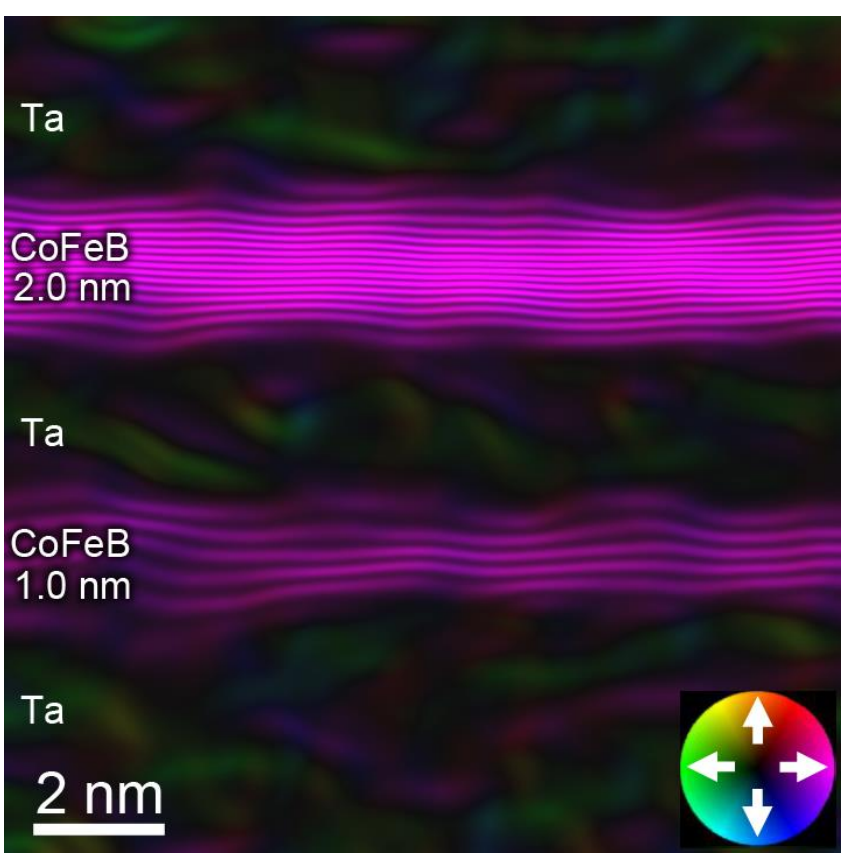

Figure 2. Magnetic flux lines in $\mathrm{CoFeB}$ layers observed by electron holography. 